\title{
The $R x$ for Change database: a first-in-class tool for optimal prescribing and medicines use
}

Michelle C Weir ${ }^{1}$, Rebecca Ryan², Alain Mayhew ${ }^{1}$, Julia Worswick', Nancy Santesso 2,3, Dianne Lowe ${ }^{2}$, Bill Leslie ${ }^{4}$, Adrienne Stevens ${ }^{1}$, Sophie Hill' ${ }^{2}$, Jeremy M Grimshaw ${ }^{1,5,6^{*}}$

\begin{abstract}
Background: Globally, suboptimal prescribing practices and medication errors are common. Guidance to health professionals and consumers alone is not sufficient to optimise behaviours, therefore strategies to promote evidence-based decision making and practice, such as decision support tools or reminders, are important. The literature in this area is growing, but is of variable quality and dispersed across sources, which makes it difficult to identify, access, and assess. To overcome these problems, by synthesizing and evaluating the data from systematic reviews, we have developed $R x$ for Change to provide a comprehensive, online database of the evidence for strategies to improve drug prescribing and use.

Methods: We use reliable and valid methods to search and screen the literature, and to appraise and analyse the evidence from relevant systematic reviews. We then present the findings in an online format which allows users to easily access pertinent information related to prescribing and medicines use. The database is a result of the collaboration between the Canadian Agency for Drugs and Technologies in Health (CADTH) and two Cochrane review groups.
\end{abstract}

Results: To capture the body of evidence on interventions to improve prescribing and medicines use, we conduct comprehensive and regular searches in multiple databases, and hand-searches of relevant journals. We screen articles to identify relevant systematic reviews, and include them if they are of moderate or high methodological quality. Two researchers screen, assess quality, and extract data on demographic details, intervention characteristics, and outcome data. We report the results of our analysis of each systematic review using a standardised quantitative and qualitative format. Rx for Change currently contains over 200 summarised reviews, structured in a multi-level format. The reviews included in the database are diverse, covering various settings, conditions, or diseases and targeting a range of professional and consumer behaviors.

Conclusions: $R x$ for Change is a novel database that synthesizes current research evidence about the effects of interventions to improve drug prescribing practices and medicines use.

\section{Background}

The safe and effective use of medicines is an important aspect of quality healthcare. While there is an abundance of data on the clinical effectiveness and safety of various drugs, this does not ensure that the drugs are being appropriately prescribed or taken; in fact, suboptimal prescribing and medication errors are common across countries [1]. Research has indicated that guidance to health professionals and consumers alone does

\footnotetext{
* Correspondence: jgrimshaw@ohri.ca

'Institute of Population Health, University of Ottawa, 1 Stewart Street,

Ottawa, ON, K1N 6N5, Canada

Full list of author information is available at the end of the article
}

not reliably change behavior, and clinical practice is often based on personal beliefs rather than on scientific evidence [2]. In order to improve professional practice, approaches that have been shown to be effective should be used to promote optimal decision making and patient care [3]. To date, a large body of evidence evaluating the effectiveness of interventions to change clinical practice has been produced $[4,5]$. However, the volume of this literature, its wide dispersion, and its variable quality make it difficult for decision makers to access, assemble, and assess this evidence [6].

To address these problems, the Canadian Optimal Medication Prescribing and Utilization Service

\section{Biomed Central}


(COMPUS) program, within the Canadian Agency for Drugs and Technologies in Health (CADTH), and in collaboration with the Cochrane Effective Practice and Organisation of Care (EPOC) Group and the Cochrane Consumers and Communication Review Group (CC\&CRG), have created and continue to update an online database of interventions to promote evidence-based prescribing and medicines use, called $R x$ for Change http://www.rxforchange.ca.

$R x$ for Change is a novel, publicly-accessible database that we initially developed and populated with reviewlevel evidence, and made available online in April 2007. We update it regularly to reflect accumulating and changing evidence and provide decision makers with reliable, up-to-date, evidence-based information in the form of reader-friendly summaries. In the database, we present key findings from systematic reviews that evaluate the effects of interventions directed at professionals, consumers, and organizations in a systematic way. This paper describes the methods for developing and populating the $R x$ for Change database and highlights key content and the significance of the database for healthcare policy makers, researchers, professionals, and consumers.

\section{Methods}

\section{Design and procedure}

Our goal for the $R x$ for Change database is to provide an overall synthesis of the evidence from systematic reviews on the effectiveness of interventions for improving prescribing by healthcare professionals and medicines use by consumers. The methods that we used to populate the database parallels systematic review methodology. We use reliable and valid methods to search and screen the literature, and to appraise and analyse the evidence from relevant systematic reviews. We then present the findings in an online format which allows users to easily access pertinent information related to prescribing and medicines use.

\section{Contributors}

In partnership with the Canadian Federal, Provincial, and Territorial Health Ministries, COMPUS identifies and promotes optimal drug therapy and encourages evidence-based information in decision making among healthcare providers and consumers. COMPUS hosts the $R x$ for Change database online in a publicly accessible format and has recruited additional funding for this project.

EPOC produces systematic reviews of interventions to improve healthcare delivery and healthcare systems, such as audit and feedback, distribution of educational materials, and decision-support tools using a well-established taxonomy of interventions and methods. In addition, EPOC has conducted overviews of existing Cochrane reviews as well as non-Cochrane systematic reviews to assess and synthesise the evidence in the area of professional behavior change [4,5]. For the $R x$ for Change database, EPOC maintains and updates the evidence from published systematic reviews on professional interventions that impact on the delivery of care, as well as organisational, financial, and regulatory interventions that influence prescribing behaviour. For the purpose of this paper, the methods used to identify and evaluate interventions targeting prescribing will be described.

The CC\&CRG produces systematic reviews of interventions targeted at consumers (patients and their family members or carers) to promote consumer participation in healthcare. The CC\&CRG has developed resources and tools to help organise and synthesise the evidence in relation to consumer communication and participation, and members of the group are currently undertaking an overview of systematic reviews of interventions directed at consumers to improve medicines use [7]. For the $R x$ for Change database, the CC\&CRG is responsible for maintaining and updating the evidence on the effect of consumer-targeted interventions, such as providing consumers with information or education on medicines use, or promoting medicines self-management skills among consumers.

Using the combined expertise of the two Cochrane review groups, we developed the methods used for the synthesis and the presentation of the findings for this database. Human resources required in successfully maintaining $R x$ for Change equals four highly trained staff members shared between both Cochrane groups, in addition to supervision from senior research staff.

\section{Results}

Identifying systematic reviews: searching and screening With the assistance of an information specialist, we conduct comprehensive regular searches of electronic databases, including MEDLINE, EMBASE, Database of Abstracts of Reviews of Effects (DARE), and the Cochrane Database of Systematic Reviews (CDSR). In addition, we systematically hand-search the CDSR and DARE databases as interventions targeting consumers' use of medicines are not well indexed.

Each year, we identify several thousand potentially eligible citations. Using explicit inclusion criteria, two researchers independently screen titles, abstracts and full text articles for relevance, and compare results. We resolve disagreements through discussion and, when necessary, through consultation with a senior team member (further details relating to selection criteria and methods are available online: http://www.cadth.ca/index.php/en/ compus/optimal-ther-resources/interventions/methods). 


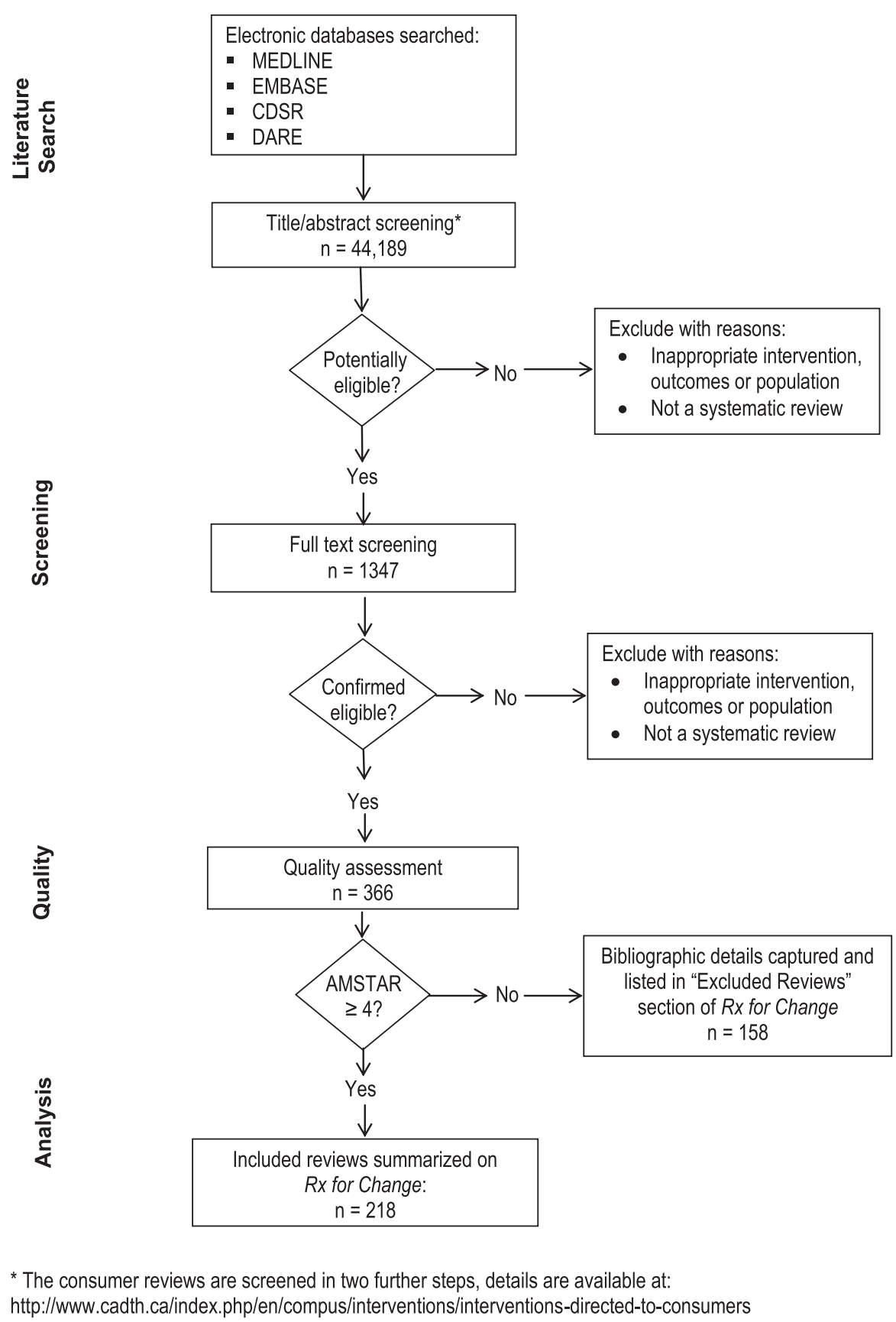

Figure 1 Flow chart describing methods and procedures of $R x$ for Change (as of April 2010).

A flow diagram illustrating the methods and procedures for the database can be found in Figure 1.

\section{Quality assessment of systematic reviews}

End users can be more confident in the results of systematic reviews that are of higher methodological quality. For this reason, two researchers critically appraise each review identified as eligible for inclusion in the database using the AMSTAR tool, a validated instrument for appraising systematic reviews [8]. AMSTAR is an 11 -item checklist on which reviews score one point for each criterion met. Items assess methodological criteria such as the comprehensiveness of the search used and whether the quality of included studies was evaluated and accounted for. In consultation with AMSTAR developers, we created decision rules for each of the 11 items to facilitate an objective and consistent assessment across reviews. 


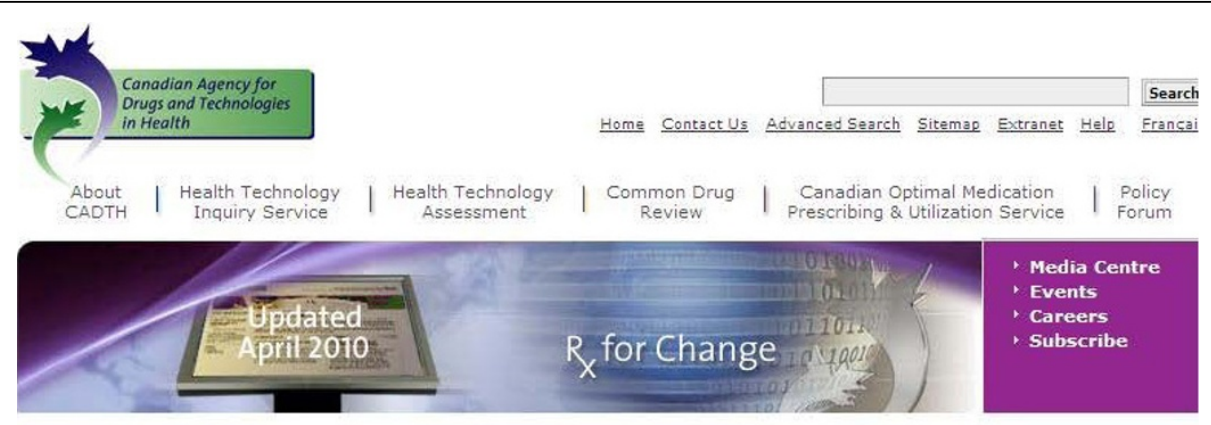

Browse " Intervention " Review " Individual studies

Browse

- Professional

Interventions that target professionals directly, aiming to improve practice.

Audit and feedback

Tailored interventions?

Distribution of educational materials ?

Educational meetings? New Evidence

Educational outreach visits

Local consensus process?

Local opinion leaders?

Mass media

Multifaceted (2) New Evidence

Patient-mediated 3 New Evidence

Professional - other 2

Reminders - computer decision support systems (drug dosing)

Reminders - computer physician order entry

Reminders - general 3 New Evidence

Consumer
Interventions that affect drug use by and prescribing for consumers.
Facilitating communication and decision making
Consumer system participation
Supporting behaviour change New Evidence
Improving quality New Evidence
Acquiring skills and competencies New Evidence
Minimising risks or harms New Evidence
Providing information or education New Evidence
Support New Evidence
Organisational
Financial
Regulatory

Figure 2 Screenshot 1 of $\boldsymbol{R} x$ for Change database. Level 1: Identifies intervention categories (professional, consumer, organizational etc.) and specific interventions (audit and feedback, acquiring skills and competencies, et al.).

Reviews are eligible to be summarised on the database if they achieve an AMSTAR score greater than 3. This decision was based on our experience that it is difficult to draw meaningful conclusions based on data from low-quality reviews. We make the bibliographic details and AMSTAR scores of these reviews available on the database under the heading 'Excluded Reviews.' To date, we have assigned approximately two-thirds of eligible reviews an AMSTAR score greater than 3, and have summarised these reviews on the database.

\section{Data extraction}

When deciding what information should be abstracted from the individual reviews, we focused on information that is useful to decision makers. Two researchers independently extract data on demographic details, 


\section{Browse " Professional: Reminders-General " Review " Individual Studies}

\section{Professional Intervention: Reminders - General}

Definition: Patient or encounter specific information, provided verbally, on paper or on a computer screen, which is designed or intended to prompt a health professional to recall information. This would usually be encountered through their general education; in the medical records or through interactions with peers, and so remind them to perform or avoid some action to aid individual patient care. Computer aided decision support is included.

Effectiveness: Four high quality reviews (Bradley, Pirkis, Shojania, Thomas) and two key reviews (Arnold, Davey) were identified. One high quality review (Shojania) found that reminder interventions were generally effective in improving both appropriate use and appropriate care outcomes. Three high quality reviews (Bradley, Thomas, Pirkis) and two key reviews (Arnold, Davey) had an insufficient number of studies to draw any conclusions about the intervention.

Summary of Overall Findings from Reviews: 1/1 high quality/key reviews with a sufficient number of studies to draw conclusions found this intervention to be generally effective.

Summary of Findings Related to Prescribing: $1 / 1$ high quality/key reviews with a sufficient number of studies to draw conclusions found this intervention to be generally effective for improving prescribing outcomes.

Bradley PM, Lindsay B. Care delivery and self-management strateqies for adults with epilepsy. Cochrane Database of Systematic Reviews 2008 (1):CD006244.

\section{9 (High)}

Pirkis JE, Jolley D, Dunt DR. Recruitment of women by GPs for pap tests: a meta-analysis. British Journal of General Practice 199848 (434):1603-1607.

\section{8 (High)}

Shojania KG, Jennings A, Mayhew A, Ramsay CR, Eccles MP, Grimshaw

J. The effects of on-screen, point of care computer reminders on processes and outcomes of care. Cochrane Database of Systematic Reviews 2009(3):CD001096.

\section{8 (High)}

Thomas L, Cullum N, McColl E, Rousseau N, Soutter J, Steen N.

Guidelines in professions allied to medicine. Cochrane Database of Systematic Reviews 2000 (1):CD000349.

\section{8 (High)}

\section{Akbari A, Mayhew A, Al-Alawi MA, Grimshaw J, Winkens R, Glidewell E,}

Figure $\mathbf{3}$ Screenshot $\mathbf{2}$ of $\boldsymbol{R} \boldsymbol{x}$ for Change database. Level 2: Provides evidence summaries within each intervention, with links to systematic review-level evidence summaries.

intervention characteristics, and outcomes from each review using a standardised data extraction form and a consensus process. This ensures a consistent summary format for each review and ensures the accuracy of the information.

\section{Analysis and synthesis}

We analyse, summarise, and report separately the results of all relevant comparisons within each systematic review using quantitative and qualitative methods as appropriate. Because reviews vary greatly in the type and amount of study data reported, we often use vote counting for data synthesis to allow for consistent presentation of results. We report our analyses by vote counting as the number of studies that favor the intervention (based on direction of effect) out of the total number of studies for each comparison. We also include any additional review data, such as metaanalyses or effect sizes. We then compile the results from each comparison and present them in a 'Table of Results.' We use standardised decision rules and statements to descriptively report on the general and medicines-specific 'Results' and 'Conclusions' of each review. For example, we use the term 'generally effective' if two-thirds or more of the studies favor the intervention. 
Clinical decision support tools for osteoporosis disease management: A systematic review of randomized controlled trials

Kastner M, Straus SE. Clinical decision support tools for osteoporosis disease management: A systematic review of randomized controlled trials. Journal of General Internal Medicine 2008 23(12):2095-2105. [PubMed]

Sources of funding: No funding. Conflict of interest not stated.

Main author affiliation: Monika Kastner, (Kastner) Department of Health Policy, Management and Evaluation, Faculty of Medicine, University of Toronto, Toronto, ON, Canada. (Straus) Department of Medicine, University of Calgary, Calgary, AB, Canada. (Kastner) Department of Health Policy, Management and Evaluation, Faculty of Medicine, University of Toronto, 155 College Street, Toronto, ON M15T 3M6, Canada.

Q: What is the effectiveness of interventions that support clinical decision making in osteoporosis disease management?

\section{BACKGROUND}

The gap between evidence and clinical practice for the diagnosis and treatment of osteoporosis has been attributed to uncertainties that relate to screening

and management of patients in clinical practice. This

review considers the effectiveness of tools that

support clinical decision making in osteoporosis

disease management for reducing fragility fractures

and increasing bone mineral density (BMD) testing or

osteoporosis therapy. Strategies include the use of

reminders (letters, guidelines in chart) as a sole

intervention or in combination with educational

training or lectures, and/or use of a risk assessment

tool. Reminders were electronic or paper-based, and

were directed at providers, patients or both.

INCLUSION

Individual studies from systematic review

SEARCH FOR EVIDENCE: Up to July 2006

INCLUDED STUDIES: 13

STUDIES RELATED TO PRESCRIBING: 7

STUDY DESIGN: RCT.

$$
\begin{aligned}
& \text { STUDY CHARACTERISTICS: } \\
& \text {, Target Population: Physicians, } \\
& \text { Pharmacists. } \\
& \text {, Intervention(s): Reminders, Multifaceted } \\
& \text { + Setting: Hospitals, Primary care } \\
& \text { practices, Communities, Not } \\
& \text { specified/unclear; Ownership - not } \\
& \text { specified/unclear; Academic - not } \\
& \text { specified/unclear. } \\
& \text {, Patients Reviewed: Age: adults, elderly; } \\
& \text { Diagnosis/clinical condition: osteoporosis, } \\
& \text { osteopenia, fracture, low BMD, premature } \\
& \text { menopause, rheumatoid arthritis. } \\
& \text { + Drug Related Outcome Categories: } \\
& \text { Appropriate use - choice. } \\
& \text {, Other Outcome Categories: Test } \\
& \text { ordering, procedures. }
\end{aligned}
$$

REVIEW QUALITY: 7
RESULTS - OVERALL

All of the included studies were RCT's $(n=8)$. Reminders $(n=5)$ and multifaceted $(n=3)$ interventions were found to be generally effective for appropriate care outcomes. The multifaceted interventions consisted of a reminder(s) plus one or more professional interventions. We excluded five studies from our analysis as they were beyond the scope of this review.

\section{RESULTS - RELATED TO PRESCRIBING}

\begin{tabular}{|c|c|c|c|c|}
\hline Comparison & Outcome & N & Analysis & Results \\
\hline \multirow[t]{2}{*}{$\begin{array}{l}\text { Reminders vs } \\
\text { control }\end{array}$} & $\begin{array}{l}\text { Appropriate } \\
\text { care }\end{array}$ & 5 & $\begin{array}{l}\text { Vote counting based on } \\
\text { direction of effect }\end{array}$ & $\begin{array}{l}\text { Vote counting } 5 / 5 \mathrm{RCT} \text { favoured } \\
\text { intervention: generally effective. }\end{array}$ \\
\hline & & & & $\begin{array}{l}\text { Prescribing related outcomes: } \\
\text { Appropriate use - Choice: } \\
\text { Vote counting } 2 / 4 \text { RCTs favoured } \\
\text { intervention: mixed effects. }\end{array}$ \\
\hline \multirow[t]{2}{*}{$\begin{array}{l}\text { Multifaceted vs } \\
\text { control }\end{array}$} & $\begin{array}{l}\text { Appropriate } \\
\text { care }\end{array}$ & 3 & $\begin{array}{l}\text { Vote counting based on } \\
\text { direction of effect }\end{array}$ & $\begin{array}{l}\text { Vote counting } 3 / 3 \text { RCTs favoured } \\
\text { intervention: generally effective. }\end{array}$ \\
\hline & & & & $\begin{array}{l}\text { Prescribing related outcomes: } \\
\text { Appropriate use - Choice: } \\
\text { Vote countina } 7 / 3 \text { RCTs favoured }\end{array}$ \\
\hline
\end{tabular}

Multifaceted $(n=3)$ interventions were found to be generally effective for appropriate use - choice outcomes while reminders $(n=4)$ reported mixed results.

CONCLUSIONS - OVERALL

Medium quality review. Reminders were found to be generally effective for appropriate care outcomes. Too few studies were located to make conclusions on the effectiveness of multifaceted interventions on appropriate care outcomes.

\section{CONCLUSIONS - RELATED TO PRESCRIBING}

Medium quality review. Relatively few studies were located to make conclusions on other reported prescribing related outcomes.

RELATED INTERVENTIONS

$$
\text { , Multifaceted }
$$

Table of Results

Figure 4 Screenshot $\mathbf{3}$ of $\boldsymbol{R x}$ for Change database. Level 3: Provides synthesis of systematic review-level evidence. 


\section{Clinical decision support tools for osteoporosis disease management: A systematic review of randomized controlled trials}

Kastner M, Straus SE. Clinical decision support tools for osteoporosis disease management: A systematic review of randomized controlled trials. Journal of General Internal Medicine 2008 23(12):2095-2105. [PubMed]

\section{Individual Studies}

Links are provided when the review can be accessed through PubMed.

1. Barr, RJ, Stewart, A, Torgerson, DJ, Seymour, DG, Reid, DM. Screening elderly women for risk of future fractures--participation rates and impact on incidence of falls and fractures. Calcified tissue international 200576 (4):243-8. [PubMed]

2. Bliuc, D, Eisman, JA, Center, JR. A randomized study of two different information-based interventions on the management of osteoporosis in minimal and moderate trauma fractures. Osteoporosis international : a journal established as result of cooperation between the European Foundation for Osteoporosis and the National Osteoporosis Foundation of the USA 200617 (9):1309-17. [PubMed]

3. Boyd, JL, Holcomb, JP, Rothenberg, RJ. Physician treatment of osteoporosis in response to heel ultrasound bone mineral density reports. Journal of clinical densitometry: the official journal of the International Society for Clinical Densitometry 20025 (4):375-81. [PubMed]

4. Devereux, K, Robertson, D, Briffa, NK. Effects of a water-based program on women 65 years and over: a randomised controlled trial. The Australian journal of physiotherapy 200551 (2):102-8. [PubMed]

5. Feldstein, A, Elmer, PJ, Smith, DH, Herson, M, Orwoll, E, Chen, C, Aickin, M, Swain, MC. Electronic medical record reminder improves osteoporosis management after a fracture: a randomized, controlled trial. Journal of the American Geriatrics Society 200654 (3):450-7. [PubMed]

6. Gardner, MJ, Brophy, RH, Demetrakopoulos, D, Koob, J, Hong, R, Rana, A, Lin, JT, Lane, JM. Interventions to improve osteoporosis treatment following hip fracture. A prospective, randomized trial. The Journal of bone and joint surgery. American volume 200587 (1):3-7. [PubMed]

7. Lacroix, AZ, Buist, DS, Brenneman, SK, Abbott, TA. Evaluation of three population-based strategies for fracture prevention: results of the osteoporosis population-based risk assessment (OPRA) trial. Medical Care 200543 (3):293-302. [PubMed]

8. MCDonough, RP, Doucette, WR, Kumbera, P, Klepser, DG. An evaluation of managing and educating patients on the risk of glucocorticoid-induced osteoporosis. Value in health : the journal of the International Society for Pharmacoeconomics and Outcomes Research 8 (1):24-31. [PubMed]

9. Morrison, LS, Tobias, JH. Effect of a case-finding strategy for osteoporosis on bisphosphonate prescribing in primary care. Osteoporosis international : a journal established as result of cooperation between the European Foundation for Osteoporosis and the National Osteoporosis Foundation of the USA 2005 16 (1):71-7. [PubMed]

10. Rolnick, SJ, Kopher, R, Jackson, J, Fischer, LR, Compo, R. What is the impact of osteoporosis education and bone mineral density testing for postmenopausal women in a managed care setting?. Menopause (New York, N.Y.) 20018 (2):141-8. [PubMed]

Figure 5 Screenshot $\mathbf{4}$ of $\boldsymbol{R} \boldsymbol{x}$ for Change database. Level 4: Provides a list of included studies within a systematic review, with links to PubMed.

We organize the reviews of interventions directed to professionals and the delivery of care using the EPOC taxonomy of interventions. This taxonomy groups interventions into five broad categories: interventions targeting healthcare professionals, changing the organization of healthcare, financial interventions, regulatory interventions, and structural changes. Each category includes a number of specific interventions. Examples of interventions targeting professionals include reminders, educational meetings, and audit and feedback. Because the consumer literature on medicines use had not been previously well organised, the CC\&CRG developed a 
Table 1 Number of systematic reviews on Rx for Change at each update

\begin{tabular}{lcc}
\hline Update & No. consumer reviews & No. professional reviews \\
\hline April 2007 & 21 & 23 \\
\hline April 2009 & 33 & 82 \\
\hline October 2009 & 53 & 124 \\
\hline April 2010 & 63 & 155 \\
\hline
\end{tabular}

taxonomy of consumer directed interventions $[9,10]$. Examples of interventions include the provision of information or education and behaviour change support. We provide definitions of each intervention on the database. For each intervention, we summarise the evidence from all of the relevant systematic reviews. We create each intervention summary based on our findings from high quality and key reviews, and this includes a statement of the overall effectiveness of the intervention and the findings as they relate to prescribing and medicines use. For those interventions where no reviews were identified, we include a comment in the database, informing the users that there is a lack of review evidence.

With each update of the database, we combine new evidence with existing evidence and intervention summaries are updated. We display flags that indicate which interventions have been recently updated with new evidence.

We present the database in a multilevel approach. In the first level, we provide a list of interventions grouped into five categories: professional, consumer, organisational, financial, and regulatory (Figure 2). In the second level, we provide intervention summaries based on the findings from high quality and key systematic reviews (Figure 3). In the third level, we provide a summary of findings from the included studies in each systematic review (Figure 4). In the fourth level, we provide links to the studies included in each systematic review (Figure 5).

\section{Implementation}

We launched the database in April 2007 and have since updated it three times (Table 1). We initially populated it with approximately 50 reviews, and it now contains over 200 summarised reviews (as of April 2010). The reviews that we have included in the database are diverse, spanning various settings, conditions, or diseases, and targeting a range of professionals, healthcare systems, and consumers. Details regarding the epidemiology and quality of reviews included in the $R x$ for Change database on professional behaviour change [11] and consumer-focused interventions [7] can be found elsewhere.

\section{Discussion}

$R x$ for Change is a well-designed database containing valuable information for researchers, healthcare providers, and policy makers. Since its inception, we have received positive feedback about the database from international users about its value, applicability, and quality. Within a year of its launch, it had accumulated more than 25,000 page views. With increasing awareness of the database and its ongoing updates, we anticipate that this interest will continue to grow. We will continue to disseminate key messages to local and international groups about which interventions are effective, and where gaps in the evidence exist. We will continue to explore methods to disseminate and translate key messages to end users, particularly as new evidence is found and added to the database.

The $R x$ for Change database has provided the opportunity for EPOC and CC\&CRG to collaborate with organizations that have strong links with healthcare decision makers (e.g., CADTH, National Prescribing Service Australia). This collaboration promotes the use of research evidence and ensures that the data is available to the general public, healthcare professionals, and policy makers.

\section{Summary}

We created the $R x$ for Change database to facilitate and improve the processes of accessing, searching, identifying, and using research to inform evidence-based prescribing and medicines use. It provides reliable, up-to-date, evidence for a wide range of users and is organised in an easy-to-browse format. We take the quality of the evidence into consideration to provide useable summaries that are relevant to decision-makers. This database is a first-in-class tool, and we will continue to promote it to ensure that it is utilized to its full potential.

\section{Acknowledgements}

The authors gratefully acknowledge the Canadian Agency for Drugs and Technologies in Health (CADTH), for making this project possible. We also thank the Canadian Institutes for Health Research, KT Canada, and the National Prescribing Service for their generous contributions to this project. Jeremy Grimshaw holds a Canada Research Chair in Health Knowledge Transfer and Uptake. We acknowledge the contributions of Doug Salzwedel, Jessie McGowan and Michelle Fiander for their work in literature searching for this project; and to Carolyn Wayne for help in editing the manuscript.

\section{Author details}

${ }^{1}$ Institute of Population Health, University of Ottawa, 1 Stewart Street, Ottawa, ON, K1N 6N5, Canada. ${ }^{2}$ Centre for Health Communication and Participation, Australian Institute for Primary Care and Ageing, La Trobe University, Melbourne, VIC, 3086, Australia. ${ }^{3}$ Department of Clinical Epidemiology and Biostatistics, McMaster University, 1200 Main Street West, Hamilton, ON, L8N 3Z5, Canada. ${ }^{4}$ Canadian Agency for Drugs and Technologies in Health, 600-865 Carling Avenue, Ottawa, ON, K1 S 5S8, Canada. ${ }^{5}$ Clinical Epidemiology Program, Ottawa Health Research Institute, 1053 Carling Avenue, Administration Building, Room 2-017, Ottawa ON, K1Y 4E9, Canada. ${ }^{6}$ Department of Medicine, University of Ottawa, Ottawa, ON, Canada.

\section{Authors' contributions}

MW participated in the design, data collection, analysis, coordination of the study, and drafted the manuscript. RR, AM, JW, NS, DL, AS, and SH

participated in the design, data collection, analysis, and coordination of the 
study and contributed to the manuscript. BL participated in the design of the study and provided feedback on the manuscript. JG conceived of the study, participated in its design and coordination and provided feedback on the manuscript. All authors read and approved the final manuscript.

\section{Competing interests}

$\mathrm{BL}$ is currently employed by CADTH. MW, AM, JW, and AS have been or are currently employed by EPOC. JG is the Coordinating Editor of EPOC. RR, NS and DL are currently employed by CC\&CRG. SH is the Coordinating Editor of CC\&CRG.

Received: 13 August 2010 Accepted: 18 November 2010

Published: 18 November 2010

\section{References}

1. Schoen C, Osborn R, Huynh PT, Doty M, Zapert M, Peugh J, al. e: Taking the pulse of health care systems: experiences of patients with health problems in six countries. Health Affairs (web exclusive) 2005, W5509-W505-525.

2. Grol R: Personal paper. Beliefs and evidence in changing clinical practice. BMJ 1997, 315(7105):418-421.

3. Grimshaw JM, Eccles MP, Walker AE, Thomas RE: Changing physicians' behavior: what works and thoughts on getting more things to work. J Contin Educ Health Prof 2002, 22(4):237-243.

4. Grimshaw JM, Shirran L, Thomas R, Mowatt G, Fraser C, Bero L, et al: Changing provider behavior: an overview of systematic reviews of interventions. Med Care 2001, 39(Suppl 2):II2-45.

5. Bero LA, Grilli R, Grimshaw JM, Harvey E, Oxman AD, Thomson MA: Closing the gap between research and practice: an overview of systematic reviews of interventions to promote the implementation of research findings. The Cochrane Effective Practice and Organization of Care Review Group. BMJ 1998, 317(7156):465-468.

6. Lavis JN, Davies HT, Gruen RL, Walshe K, Farquhar CM: Working within and beyond the Cochrane Collaboration to make systematic reviews more useful to healthcare managers and policy makers. Healthc Policy 2006, 1(2):21-33.

7. Ryan R, Santesso N, Hill S, Kaufman C, Grimshaw J: Consumer-oriented interventions for evidence based prescribing and medicine use: an overview of Cochrane reviews. Cochrane Database of Systematic Reviews 2009, , 2: CD007768.

8. Shea BJ, Bouter LM, Peterson J, Boers M, Andersson N, Ortiz Z, Ramsay T, Bai A, Shukla VK, Grimshaw JM: External validation of a measurement tool to assess systematic reviews (AMSTAR). PLoS One 2007, 2(12):e1350.

9. Ryan R, Lowe D, Santesso N, Hill S: Development of a taxonomy of interventions directed at consumers to promote evidence-based prescribing and medicines use: a tool for evidence-based decisionmaking (Poster session 3: Safe and effective use). National Medicines Symposium: 26-28 May 2010; Melbourne 2010 [http://www.nms2010.org.au/ pdf/nps_nms_2010abstracts.pdf].

10. Lowe D, Ryan R, Santesso N, Hill S: Development of a taxonomy of interventions to organise the evidence on consumers' medicines use. Patient Education and Counseling 2010.

11. Weir M, Mayhew A, Worswick JFD, Grimshaw J: The Epidemiology and Quality of Systematic Reviews of Health Professional Behaviour Change Interventions. 17th Cochrane Colloquium: 11-14 October 2009; Singapore 2009 [http://www.imbi.uni-freiburg.de/OJS/cca/index.php/cca/article/view/ 8042].

doi:10.1186/1748-5908-5-89

Cite this article as: Weir et al:: The Rx for Change database: a first-inclass tool for optimal prescribing and medicines use. Implementation Science 2010 5:89.

\section{Submit your next manuscript to BioMed Central and take full advantage of:}

- Convenient online submission

- Thorough peer review

- No space constraints or color figure charges

- Immediate publication on acceptance

- Inclusion in PubMed, CAS, Scopus and Google Scholar

- Research which is freely available for redistribution

Submit your manuscript at www.biomedcentral.com/submit
Biomed Central 